\title{
Switching Flow-Graph Nonlinear Modeling Technique
}

\author{
Keyue Smedley, Member, IEEE, and Slobodan Cuk, Member, IEEE
}

\begin{abstract}
A unified graphical modeling technique, "Switching Flow-Graph" is developed to study the nonlinear dynamic behavior of pulse-width-modulated (PWM) switching converters. Switching converters are variable structure systems with linear subsystems. Each subsystem can be represented by a flow-graph. The Switching Flow-Graph is obtained by combining the flowgraphs of the subsystems through the use of switching branches. The Switching Flow-Graph model is easy to derive, and it provides a visual representation of a switching converter system. Experiments demonstrate that the Switching Flow-Graph model has very good accuracy.
\end{abstract}

\section{INTRODUCTION}

$\mathbf{S}$ WITCHING converters are pulsed and nonlinear dynamic systems. Such a system may be stable in the vicinity of the operating point; however, if the system is perturbed enough to cross a separatrix, it may never return to its operating point. A small-signal model does not predict the stability information when the system is subject to large perturbations. A largesignal modeling tool is necessary to study the global dynamic behavior of switching converters and to design robust systems. There have been efforts in the past to model large-signal dynamics [2], [3]. [2] provides a large-signal trajectory study for sliding mode converter systems only. The model presented in [3] is in two forms, a discrete-time equation for use in computer simulations and a continuous-time matrix expression for physical understanding. Since the model is in a matrix form, discrete-time or continuous-time, the cause-and-effect relationship between the circuit parameters and the circuit dynamics are not very obvious. Those models [2], [3] do not provide a unified general form for large-signal, small-signal, and steady-state analysis.

In this paper a general graphic nonlinear modeling tool, the Switching Flow-Graph technique, is developed to reveal the global and local dynamic properties of switching converters. This technique utilizes the state space averaging concept [1] and is an extension of linear circuit flow-graph theory. The proposed modeling tool is a graphic modeling tool, and for the first time clearly presents the cause and effect of the large-signal dynamics of the switching converters, which gives directions for parameter adjustment in the engineering design. The proposed modeling tool provides unified large-signal, steady-state, and small-signal models for switching converters,

Manuscript received March 17, 1993; revised April 18, 1994.

Keyue Smedley is currently with the Department of Electrical Engineering, University of California, Irvine, CA 92717 USA

Slobodan Cuk is with the Power Electronics Group, California Institute of Technology, Pasadena, CA 91125 USA.

IEEE Log Number 9404417. which is significantly advanced from the existing modeling tools.

Though a switching converter is overall a nonlinear system, it is equivalent to a linear circuit, the $\mathrm{ON}$-circuit, when the switch is in the ON state, and it is equivalent to another linear circuit, the OFF-circuit, when the switch is in the OFF state. The ON-circuit and the OFF-circuit can be described by their respective flow-graphs. The Switching Flow-Graph is obtained by combining the flow-graphs of the ON-circuit and the OFFcircuit through the use of switching branches. The switching branches are the only nonlinear components in the Switching Flow-Graph; therefore, the modeling work is reduced to the switching branches. The Switching Flow-Graph technique is very easy to use, and it provides a visual understanding of switching converter systems. It can predict large-signal, small-signal, and steady-state behavior of switching converter systems. The result of the small-signal model is identical to the state-space averaging method, the result of the largesignal model has been verified by experiments. This technique is described in Section II. In Section III, a buck converter with input filter was built to verify the Switching Flow-Graph technique. Conclusion and discussions are given in Section IV.

\section{SWITCHING FLOW-GRAPH MODEL}

Flow-graph is a symbolic language for the description of linear dynamic systems that provides a graphic representation of the signal flow within the system. This language is extended to describe the nonlinear dynamics of switching converters.

\section{Flow-Graph Background}

The signals are represented by nodes, which are schematically represented by small circles in the diagrams. The nodes are connected by branches, line segments with arrows. The signals flow only in the direction of the arrow on each branch. Each branch has a transmittance or gain indicated next to it and the signals passing along that branch are multiplied by the branch transmittance. The signal at a node is the sum of all signals entering the node. Input nodes, nodes with output branches only, represent independent variables. A normal node has both inputs and outputs, but can be considered as an output node. A path is any collection of a continuous succession of branches traversed in the same direction. A forward path is a path that starts at an input node and ends at an output node, along which no node is traversed more than once. A loop is a path that originates and terminates on the same node, and along which no other node is encountered more than once. 


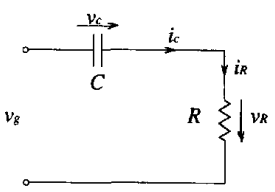

Fig. 1. The RC circuit.

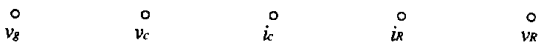

(a)

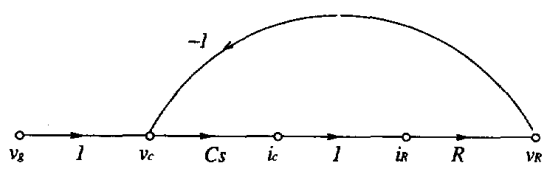

(b)

Fig. 2. (a) Five nodes of the RC circuit. (b) The flow-graph of the RC circuit.

The procedure for constructing the flow-graph of a system is straightforward. First each variable of interest is assigned a node on the flow-graph. Next the nodes are interconnected as required by the specific system configuration. Once all the nodes are properly connected, the flow-graph is complete.

Consider a simple $R C$ circuit as an example as shown in Fig. 1, where $v_{g}$ is the input voltage, $v_{C}$ is the voltage across the capacitor, $i_{C}$ is the capacitor current, $i_{R}$ is the resistor current, and $v_{R}$ is the output voltage. To create the flow-graph for this RC circuit, first assign five nodes from left to right to denote $v_{g}, v_{C}, i_{C}, i_{R}$, and $v_{R}$, respectively, as shown in Fig. 2 (a). From Kirchhoff's law, it is found that:

$$
\begin{aligned}
v_{C} & =v_{g}-v_{R} \\
i_{C} & =C s v_{C} \\
i_{R} & =i_{C} \\
v_{R} & =R i_{R},
\end{aligned}
$$

where $C$ is the capacitance, $R$ is the resistance, and $s$ is the Laplace complex number $s=\sigma+j \omega$. These relations are used to connect the nodes in Fig. 2 (a), to produce the flow-graph shown in Fig. 2 (b).

The node that represents the input voltage $v_{g}$ is an input node, while the other nodes are normal nodes. $v_{R}$ is also considered as an output node. The path from $v_{g}$ to $v_{R}$ is a forward path. The path originating from node $v_{c}$, traversing $i_{c}$, $i_{R}, v_{R}$, and ending at node $v_{c}$ forms a loop. From the flowgraph, the input to output transfer function can be obtained for the circuit:

$$
\begin{aligned}
\frac{v_{R}}{v_{g}} & =\frac{\text { ForwardGain }}{1-\text { LoopGain }} \\
& =\frac{R C s}{1+R C s} .
\end{aligned}
$$

To derive transfer functions for more complicated circuits, Flow-graph algebraic rules or Mason's formula [4] are recommended.

\section{Switching Flow-Graph}

Switching converters are nonlinear dynamic systems; however, deeper insight reveals that switching converters are systems with variable structure. A switching converter, in continuous mode, contains two linear subcircuits. The subcircuits share common elements, such as inductors, capacitors, and resistors, connected in different topologies. The switch of the converter operates at a frequency, either constant or variable, and alters the system between the two linear subcircuits. This conceptual view provoked the motivation to model these two linear subcircuits using flow-graphs, then to relate the flowgraphs to each other using switching branches.

Suppose a switching converter operates at a frequency $f_{s}(t)=\frac{1}{T_{s}(t)}$. When $0<t<T_{O N}$, switch $S$ is ON, and when $T_{O N}<t<T_{s}$, switch $S$ is OFF. For the two positions of the switch $S$ in the switching converter, the two switched subcircuits are obtained. Clearly a change in the topological structure occurs within each period as the circuit configuration is periodically changed from the ON-circuit to the OFF-circuit. Both switched subcircuits are linear by themselves, while the converter is a nonlinear circuit due to the periodic structure change.

During the time when the switch $S$ is in the ON position, $0<t<T_{O N}$, the converter is switched to the ON-circuit. Nodes are assigned to each variable and are connected to form a flow-graph $\mathcal{G}_{O N}$ according to the ON-circuit configuration. During the time when the switch $S$ is in the OFF position, $T_{O N}<t<T_{s}$, the converter is switched to the OFF-circuit. The OFF-circuit contains the same circuit elements as those in the ON-circuit; therefore, it preserves the same variables. The same nodes, which were used for the ON-circuit, are used to represent the OFF-circuit variables. However, the nodes are connected according to the OFF-circuit configuration to form a flow-graph $\mathcal{G}_{O F F}$.

The two flow-graphs, $\mathcal{G}_{O N}$ and $\mathcal{G}_{O F F}$, have exactly the same nodes. However, some branches that exist in $\mathcal{G}_{O N}$ may not exist in $\mathcal{G}_{O F F}$, and some branches that exist in $\mathcal{G}_{O F F}$ may not exist in $\mathcal{G}_{O N}$. The two flow-graphs $\mathcal{G}_{O N}$ and $\mathcal{G}_{O F F}$ can be topologically merged by the following equation:

$$
\mathcal{G}=k \mathcal{G}_{\text {ON }}+\bar{k} \mathcal{G}_{\text {OFF }},
$$

where $k$ and $\bar{k}$ are two complementary switching functions.

$$
\begin{aligned}
& k=\left\{\begin{array}{ll}
1 & 0<t<T_{\text {on }} \\
0 & T_{\text {ON }}<t<T
\end{array},\right. \\
& \bar{k}=\left\{\begin{array}{ll}
0 & 0<t<T_{\text {on }} \\
1 & T_{\text {ON }}<t<T
\end{array} .\right.
\end{aligned}
$$

Two switching branches, the $k$-branch and its complementary $\bar{k}$-branch, are thus introduced, as shown in Fig. 3. The transmittances of the two branches are time dependent. During the time $0<t<T_{O N}$, the $k$-branch has a transmittance of " 1, " and the $\bar{k}$-branch has a transmittance of " 0 ." During the time $T_{O N}<t<T_{S}$, the $k$ branch has a transmittance of " 0 ," and the $\bar{k}$-branch has a transmittance of " 1 ." These switching branches graphically unite the two flow-graphs, $\mathcal{G}_{O N}$ and $\mathcal{G}_{O F F}$, into one flow-graph $\mathcal{G} . \mathcal{G}$ is the graphical representation of the switching converter. The flow-graph $\mathcal{G}$ contains 


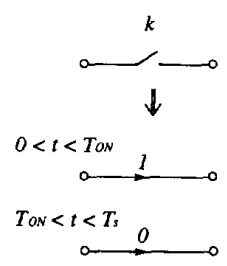

(a)

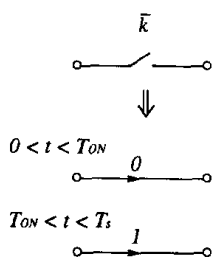

(b)

Fig. 3. The switching branches: (a) $k$ and (b) $\bar{k}$.

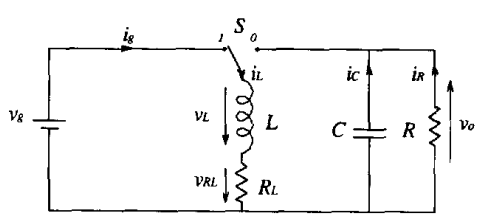

(a)
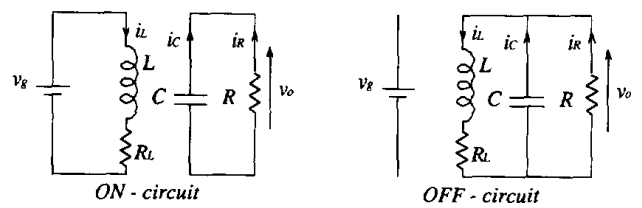

(b)

Fig. 4. (a) The buck-boost converter. (b) Its subcircuits.

switching branches, the $k$-branch and the $\bar{k}$-branch; therefore, it is defined as a Switching Flow-Graph . The switching control signals are injected into the converter through these switching branches.

Consider the buck-boost converter, shown in Fig. 4 (a), as an example, where $v_{g}$ is the input voltage, $L$ is the inductor, $C$ is the capacitor, $R$ is the load, $R_{L}$ is the parasitic resistance of the inductor, and $S$ is the switch. Define $v_{L}, v_{R_{L}}$, and $v_{o}$ as the inductor voltage, the parasitic-resistor voltage, and the output voltage respectively. Define $i_{g}, i_{L}$, and $i_{o}$ as the input current, the inductor current, and the output current flowing in the same direction as $v_{g}, v_{L}$, and $v_{o}$ respectively. The quantity $i_{o}$ is the sum of the load current and the capacitor current $i_{C}$. The two subcircuits, the ON-circuit and the OFF-circuit, are shown in Fig. 4 (b). When the switch is $\mathrm{ON}$, the buck-boost converter is equivalent to its ON-circuit, and when the switch is OFF, it is equivalent to its OFF-circuit.

The two subcircuits can be described by their flow-graphs, $\mathcal{G}_{O N}$ and $\mathcal{G}_{O F F}$, shown in Fig. 5 (a). These two flow-graphs share all of the nodes and part of the branches. By overlapping $\mathcal{G}_{O N}$ and $\mathcal{G}_{O F F}$, it is easily seen that some branches exist in both $\mathcal{G}_{O N}$ and $\mathcal{G}_{O F F}$, while other branches exist only in one of them. Branches that exist in $\mathcal{G}_{O N}$ but not in $\mathcal{G}_{O F F}$ are replaced by $k$-branches, and the branches that exist in $\mathcal{G}_{O F F}$ but not in $\mathcal{G}_{O N}$ are replaced by $\bar{k}$-branches. Therefore, $\mathcal{G}_{O N}$ and $\mathcal{G}_{O F F}$ are combined to form one Switching Flow-Graph $\mathcal{G}$, shown in Fig. 5 (b). In addition, the Switching Flow-Graph $\mathcal{G}$ of the buck-boost converter can be simplified using the algebraic rules as shown in Fig. 6.

The Switching Flow-Graph $\mathcal{G}$ is linear except for the switching branches. The switching branches are the signal-flow repre-

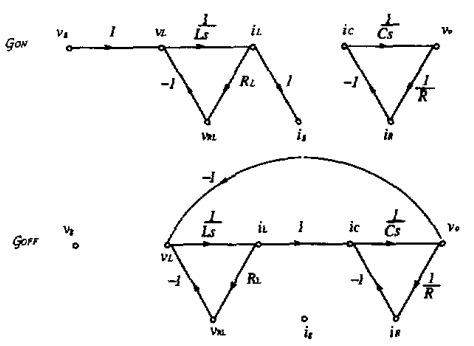

(a)

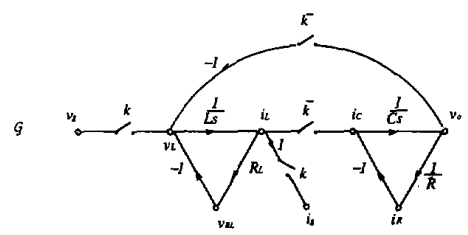

(b)

Fig. 5. (a) The Switching Flow-Graph of the subcircuits. (b) The Switching Flow-Graph of the buck-boost converter.

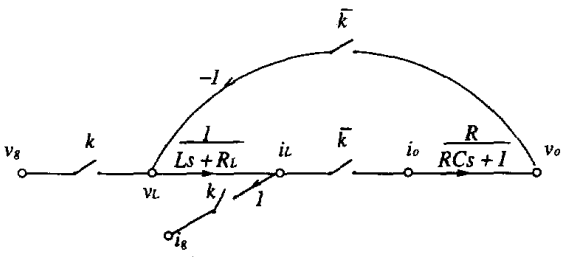

Fig. 6. The simplified Switching Flow-Graph of the buck-boost converter.

sentations of the real switches. The real switches in switching converters could have very different behavior depending on their voltage, current, and load conditions; therefore, it is very difficult to make a general model for the real switches. When switching converters are presented by Switching FlowGraphs, the switches are transferred into generalized switching branches: $k$-branches and $\bar{k}$-branches. Unified models for switching branches are easy to obtain.

\section{Large-Signal Model}

Suppose that the signal entering the $k$-branch is $x(t)$, and that the output signal of the switching branch is $y(t)$. The input signal $x(t)$ is chopped by the switching function, as shown in Fig. 7. The output signal $y(t)$ of the switching branch is a chopped waveform with an envelope equal to the input signal $x(t)$, a pulse frequency equal to the switching frequency $f_{8}$, and a pulse width equal to $T_{O N}$.

Assume that the small ripple condition is satisfied; therefore, the filter corner frequency is much smaller than the switching frequency. The effective signal carried at the output of $k$ branch is equal to its average value over a switching cycle:

$$
\begin{aligned}
y(t) & =\frac{1}{T_{s}(t)} \int_{0}^{T_{O N}(t)} x(t) d t \\
& \approx x(t) \frac{1}{T_{s}(t)} \int_{0}^{T_{O N}(t)} d t \\
& =x(t) d(t) .
\end{aligned}
$$




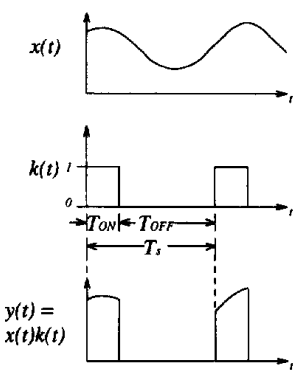

(a)

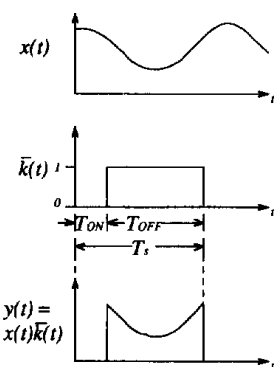

(b)
Fig. 7. (a) The signals of the k-branch. (b) The signals of the $\bar{k}$-branch.

Similarly for the $\bar{k}$-branch, the input signal $x(t)$, and output signal $y(t)$ have the relation:

$$
\begin{aligned}
y(t) & =\frac{1}{T_{s}(t)} \int_{T_{O N}(t)}^{T_{S}(t)} x(t) d t \\
& \approx x(t) \frac{1}{T_{s}(t)} \int_{T_{O N}(t)}^{T_{s}(t)} d t \\
& =x(t) d^{\prime}(t),
\end{aligned}
$$

where $d(t)$ and $d^{\prime}(t)$ are the averages of the switching functions $k(t)$ and $\bar{k}(t)$, respectively, and they represent the duty-ratio of the switch.

$$
\begin{aligned}
d(t) & =\frac{T_{O N}(t)}{T_{s}(t)} \\
d^{\prime}(t) & =\frac{T_{O F F}(t)}{T_{s}(t)} \\
d(t)+d^{\prime}(t) & =1 .
\end{aligned}
$$

Switches are controlled by their duty-ratio function. Equations (11) and (14) indicate that the output signal $y(t)$ from the switching branches is the product of the input signal $x(t)$ and the duty-ratio control signal $d(t)$ or $d^{\prime}(t)$, thus it is directly affected by the input signal $x(t)$ and controlled by the duty-ratio $d(t)$ or $d^{\prime}(t)$. Therefore, the large-signal model of the switching branches is represented by a single multiplier, as shown in Fig. 8. The large-signal model of a switching converter is obtained by replacing the switching branches with the large-signal models of the switching branches in the Switching Flow-Graph $\mathcal{G}$.

The large-signal model is shown in Fig. 9 for the same buck-boost converter discussed in the previous section. This model can be directly entered to the TUTSIM [5] simulation program to simulate the circuit dynamics. The input to output large-signal response can be obtained by injecting a signal into node $v_{g}$, and observing the output at the node $v_{o}$. In addition, the input impedance can be determined by detecting the signal at node $i_{g}$. Injecting a signal into node $d(t)$ and detecting the output at node $v_{o}$ generates the control to output large-signalresponse. Injecting a signal into the node $v_{o}$ and detecting the signal at node $i_{o}$ yields the output impedance.

It is very easy to obtain the global view of the dynamic behavior of the system by entering the model into a computer

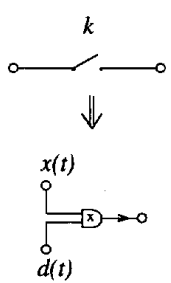

(a)

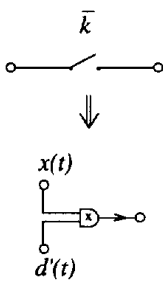

(b)

$$
\text { where } \underset{f}{[} \rightarrow \rightarrow \text { is a multiplier }
$$

Fig. 8. (a) The large-signal model of the $k$-branch. (b) The large-signal model of the $\bar{k}$-branch.

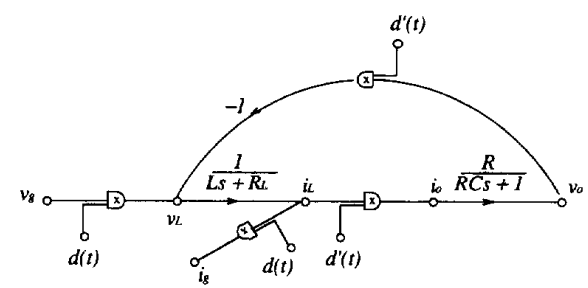

Fig. 9. The large-signal model of the buck-boost converter.

simulation program. From the study of the large-signal dynamic behavior, a desired stable operating region can be found. Inside this region, the system can be linearized in the vicinity of the operating point to obtain the small-signal frequency response.

\section{Steady-State Model}

The large-signal models of the $k$-branch and the $\bar{k}$-branch presented in the previous section can be modified to generate a steady-state model of the switching branches. Assume that the input signals and the duty-ratio control signals, $x(t), d(t)$, and $d^{\prime}(t)$, entering the switching branches are constant. That is:

$$
\begin{aligned}
x(t) & =X \\
d(t) & =D \\
d^{\prime}(t) & =D^{\prime} .
\end{aligned}
$$

Then the output signal $y(t)$ of the switch is also constant:

$$
y(t)=Y \text {. }
$$

Thus, the switching branches degenerate to standard branches, as shown in Fig. 10. In the steady-state, the $k$-branch has only one input signal, $X$; the transmittance of the branch is $D$ and the output signal is $Y$. Similarly, for the $\bar{k}$-branch, the input signal is $X$, the transmittance of the branch is $D^{\prime}$ and the output signal is $Y$.

Substitution of these steady-state models into the Switching Flow-Graph $\mathcal{G}$ of the switching converter for the switching branches, and assuming $S \rightarrow 0$, immediately yields the steadystate model of the switching converter. The steady-state model of the buck-boost converter is shown in Fig. 11.

Relations between state variables, such as the input-tooutput gain, the dc relations between the state variables, and 

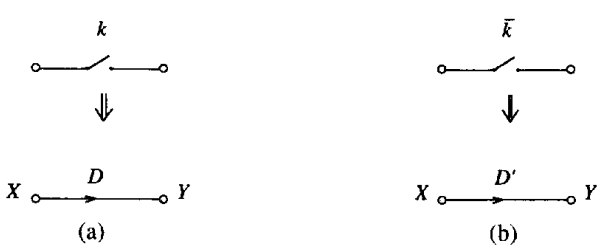

Fig. 10. (a) The steady-state models of the $k$-branch. (b) The steady-state models of the $\bar{k}$-branch.

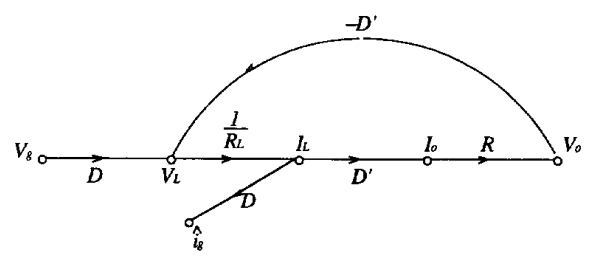

Fig. 11. The steady-state model of the buck-boost converter.

the efficiency, etc., can be found directly off the steady-state flow-graph model.

$$
\begin{aligned}
\frac{V_{o}}{V_{g}} & =\frac{D / D^{\prime}}{1+\frac{R_{L}}{R D^{\prime 2}}} \\
\frac{I_{L}}{V_{g}} & =\frac{D}{R_{L}+D^{\prime 2} R} \\
\frac{I_{C}}{V_{g}} & =\frac{D D^{\prime}}{R_{L}+D^{\prime 2} R} \\
\eta & =\frac{1}{1+\frac{R_{L}}{D^{\prime 2} R}} .
\end{aligned}
$$

\section{Small-Signal Model}

For the $k$-branch and the $\bar{k}$-branch, in the large-signal environment, the input and output signals have the relationships:

$$
\begin{array}{ll}
y(t)=x(t) d(t) & \text { for the } k \text {-branch, } \\
y(t)=x(t) d^{\prime}(t) & \text { for the } \bar{k} \text {-branch. }
\end{array}
$$

Small perturbations, $\hat{x}(t), \hat{d}(t)$, and $\hat{y}(t)$, are introduced near the operating point $X, D$ and $Y$.

$$
\begin{aligned}
d(t) & =D+\hat{d}(t) \\
d^{\prime}(t) & =D^{\prime}-\hat{d}(t) \\
1 & =D+D^{\prime} \\
x(t) & =X+\hat{x}(t) \\
y(t) & =Y+\hat{y}(t) .
\end{aligned}
$$

Inserting Equations (28), (29), (31) and (32) into the largesignal switching branch relations (26) and (27), yields the equations for the small-signal perturbations.

$$
\begin{aligned}
Y+\hat{y}(t) & =(X+\hat{x}(t))(D+\hat{d}(t)) \\
& =X D+D \hat{x}(t)+X \hat{d}(t)+\hat{x}(t) \hat{d}(t)
\end{aligned}
$$

for the $k$-branch and

$$
\begin{aligned}
Y+\hat{y}(t) & =(X+\hat{x}(t))\left(D^{\prime}-\hat{d}(t)\right) \\
& =X D^{\prime}+D^{\prime} \hat{x}(t)-X \hat{d}(t)-\hat{x}(t) \hat{d}(t)
\end{aligned}
$$

for the $\bar{k}$-branch.

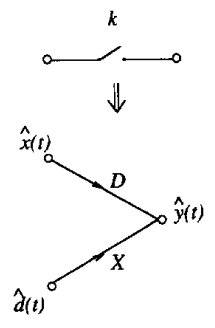

(a)

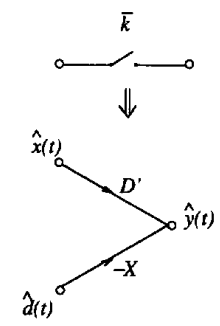

(b)
Fig. 12. (a) The small-signal models of the $k$-branch. (b) The small-signal models of the $\bar{k}$-branch.

At the operating point, the $k$-branch and the $\bar{k}$-branch satisfy:

$$
\begin{array}{ll}
Y=X D & \text { for the } k \text {-branch, } \\
Y=X D^{\prime} & \text { for the } \bar{k} \text {-branch. }
\end{array}
$$

Neglecting the second-order perturbations, yields the smallsignal switching branch equations:

$$
\begin{array}{ll}
\hat{y}(t)=D \hat{x}(t)+X \hat{d}(t) & \text { for the } k \text {-branch, } \\
\hat{y}(t)=D^{\prime} \hat{x}(t)-X \hat{d}(t) & \text { for the } \bar{k} \text {-branch. }
\end{array}
$$

The small-signal models of the switching branches are shown in Fig. 12. Substitution of the small-signal models for the switching branches in the Switching Flow-Graph immediately generates the small-signal model.

The small-signal dynamic model is shown in Fig. 13 for the same buck-boost converter discussed in the last section. It can be further simplified using the flow-graph algebraic rules. First move all the branches with input $\hat{d}$ to one node, for example node $v_{L}$. The branch with gain $-V_{o}$ is moved forward though the branch with gain -1 ; therefore, the moved branch will have a new gain $-V_{o} \times(-1)=V_{o}$. The branch with gain $-I_{L}$ is moved backward though branch with gain $D^{\prime}$ and branch with gain $\frac{1}{L_{s}+R_{L}}$; therefore, the moved branch will have a new gain $\frac{-1\left(L s+R_{L}\right) I_{L}}{D^{\prime}}$. Now all the three branches with input $\hat{d}$ are connected in the node as shown in Fig. 14, which results a combined new branch with gain $V_{g}+V_{o}-\frac{\left(L s+R_{L}\right) I_{L}}{D^{\prime}}$ as shown in Fig. 15. The analytic forms of the transfer functions for the buck-boost converter are obtained from Fig. 15.

$$
\begin{aligned}
& \frac{\hat{v}_{o}}{\hat{d}}=\frac{V_{g}+V_{o}-\frac{L s+R_{L}}{D^{\prime}} I_{L}}{D^{\prime}\left(\left(1+\frac{R_{L} / R}{D^{\prime 2}}\right)+\frac{R_{L} C+L / R}{D^{\prime 2}} s+\frac{L C}{D^{\prime 2}} s^{2}\right)} \\
& \frac{\hat{v}_{o}}{\hat{v}_{g}}=\frac{\frac{D}{D^{\prime}}}{\left(1+\frac{R_{L} / R}{D^{\prime 2}}\right)+\frac{R_{L} C+L / R}{D^{\prime 2}} s+\frac{L C}{D^{\prime 2}} s^{2}} .
\end{aligned}
$$

The transfer function, shown in Equation (39), which represents the relationship between the small-signal control $\hat{d}(t)$ and the output $\hat{v}_{o}$, can be simplified using the steady-state relations of Equation (22) and (23).

$$
\frac{\hat{v}_{o}}{\hat{d}}=\frac{V_{g}\left(1+\frac{D\left(R D^{\prime}-R_{L} / D^{\prime}\right)}{R_{L}+R D^{\prime 2}}-\frac{L D / D^{\prime}}{R_{L}+R D^{\prime 2}} s\right)}{D^{\prime}\left(\left(1+\frac{R_{L} / R}{D^{\prime 2}}\right)+\frac{R_{L} C+L / R}{D^{\prime 2}} s+\frac{L C}{D^{\prime 2}} s^{2}\right)} .
$$




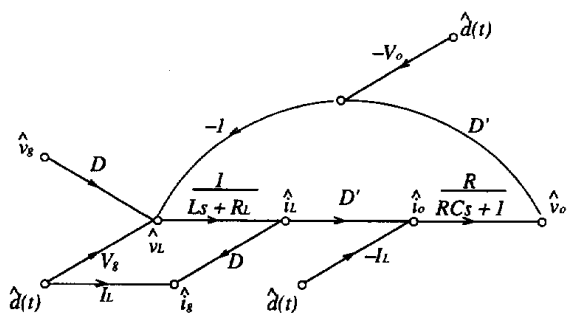

Fig. 13. The small-signal model of the buck-boost converter.

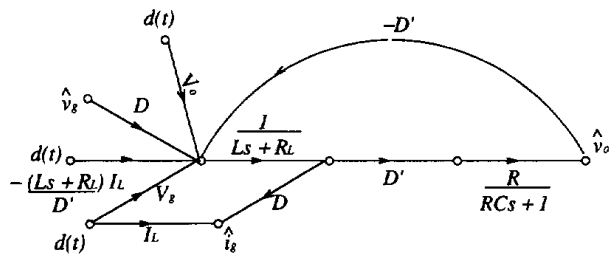

Fig. 14. Simplification of the small-signal model of the buck-boost converter using algebraic rules.

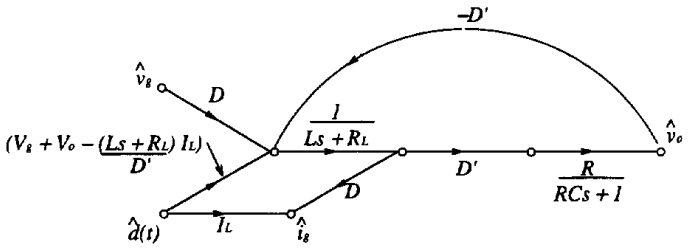

Fig. 15. The simplified small-signal model of the buck-boost converter.

The small-signal transfer functions, obtained using the Switching Flow-Graph technique, are the same as those obtained using the state-space averaging technique; however, the Switching Flow-Graph technique provides a more visible and faster way to obtain the small-signal models for switching converters. The Switching Flow-Graph models generate a simple representation of the signal processing in switching converters.

In addition, the Switching Flow-Graph facilitates the determination of the input and output impedance:

$$
\begin{aligned}
\hat{z}_{i n} & =\frac{\hat{v}_{g}}{\hat{i}_{g}} \\
& =\frac{R \frac{D^{\prime 2}}{D^{2}}\left(1+\frac{R_{L} / R}{D^{2}}+\frac{R_{L} C+L / R}{D^{\prime 2}} s+\frac{L C}{D^{\prime 2}} s^{2}\right)}{1+R C s} \\
\hat{z}_{\text {out }} & =\frac{\hat{v}_{o}}{\left.\hat{i}_{o}\right|_{v_{g}=0}} \\
& =\frac{R_{L}\left(1+\frac{L}{R_{L}} s\right)}{D^{\prime 2}\left(\left(1+\frac{R_{L} / R}{D^{2}}\right)+\frac{R_{L} C+L / R}{D^{\prime 2}} s+\frac{L C}{D^{\prime 2}} s^{2}\right)} .
\end{aligned}
$$

The Switching Flow-Graph technique is an easily implemented graphic modeling tool for switching converter design and analysis. This technique provides a large-signal model, a small-signal model, and a steady-state model. The largesignal model gives a global view of the switching converter, thus it can be used to find the convergent operating region and to design a converter that operates in a desired region.

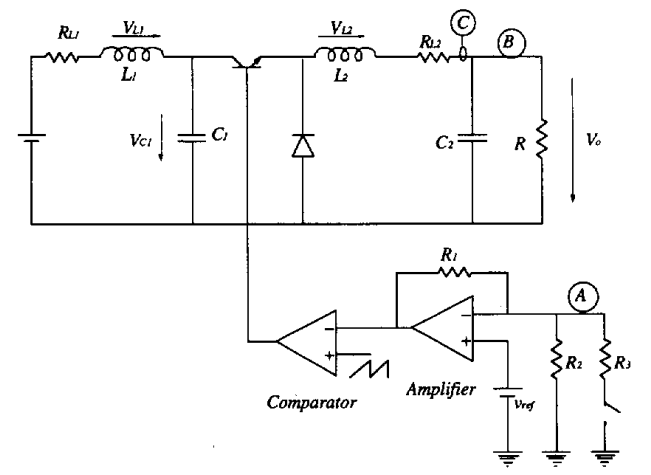

Fig. 16. The experimental buck converter with an input filter.

The steady-state model yields the steady-state relations, which are useful in determining the efficiency and other steadystate properties of switching converters. The small-signal model gives the transfer functions from one arbitrary state variable to another arbitrary state variable, such as the inputto-output gain, the control-to-output gain, the input and output impedanice, etc.

\section{EXPERIMENTAL VERIFICATION}

The Switching Flow-Graph is a graphic presentation of the circuit that contains complete dynamic information about the circuit, provides physical insight into the operation of the circuit and is very easy to obtain. Experiments were conducted to verify Switching Flow-Graph technique.

\section{Experimental Circuit}

A buck converter has a linear second-order relation from its control to output when there is no input filter. However, the dynamic behavior of the buck converter is dramatically changed by the existence of an additional input filter. The system becomes nonlinear and right half plane (RHP) zeros may appear in its sjmall-signal transfer function. Thus, a buck converter with an input filter, as shown in Fig. 16, was chosen for the experiments. $L_{1}$ and $L_{2}$ are the input and output inductors, $C_{1}$ and $C_{2}$ are the input and output capacitors, $R_{L 1}$ and $R_{L 2}$ are the parasitic resistance, and $R$ is the load resistance. Define $v_{g}$ as the input voltage, $v_{L 1}$ and $v_{L 2}$ as the voltages across $L_{1}$ and $L_{2}, v_{C 1}$ as the voltage across $C_{1}$, and $v_{o}$ as the output voltage. Define $i_{g}$ as the input current, $i_{L 1}$ and $i_{L 2}$ as the currents through $L_{1}$ and $L_{2}, i_{C 1}$ as the current through $C_{1}$, and $i_{o}=i_{L 2}=i_{R}+i_{C 2}$ as the output current. All currents flow in the same direction as their associated voltages.

\section{Large-Signal Model}

The large-signal model for the buck converter with input filter is shown in Fig. 17. To verify the large-signal model, the results obtained from computer simulations were compared to the results obtained from experiments. The commercially available simulation program, TUTSIM, is flow-graph oriented. The large-signal model, as shown in Fig. 17, can be 


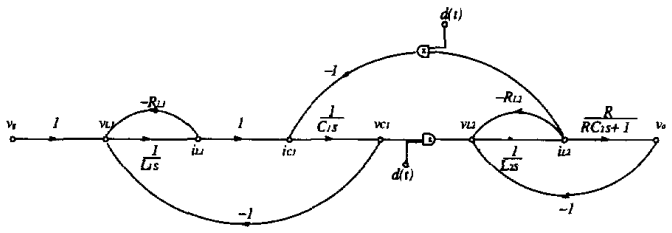

Fig. 17. The large-signal model of the buck converter with an input filter

$$
\begin{aligned}
& \text { Model File: bfstp } 596 . c 10 \\
& \text { Date: } \quad 4 / 22 / 1990 \\
& \text { Timing: } 300.000 E-09 \text {,DELTA ; } 0.0050000 \text {,RANGE } \\
& \text { PlotBlocks } \\
& \text { Forma } \\
& \text { Horz: } \begin{array}{ccc}
\text { BlockNo, Plot-MiNimum, Plot-MAXimum; Comment } \\
\text { Y } & 0.0000,0.0050000 ; \text {; Time }
\end{array} \\
& \text { Y1: } \quad 12, \quad 0.0000, \quad 20.0000
\end{aligned}
$$

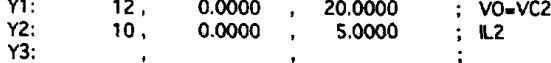

$$
\begin{aligned}
& \text { Y4: ; ; ; } \\
& 15.0000 \quad 1 \mathrm{CON} \\
& 0.0010000 \quad 3 \text { PLS } \quad \text {;D_detta } \\
& 0.0100000 \\
& \text { 430.000E-06 } \\
& \begin{array}{lrrl}
4 \text { SUM } & 2 & 3 & \text {;D_O+D_delta } \\
5 \mathrm{MUL} & 4 & 10 & \text {;PWM SWITCH }
\end{array} \\
& \begin{array}{lllll}
6 \mathrm{~L} & 1 & -8 & -7 & ; 1 \mathrm{~L} 2
\end{array} \\
& \begin{array}{lllll}
0.2700000 & 7 R & 6 & \text {;R11 }
\end{array} \\
& \begin{array}{lllll}
10.400 \mathrm{E}-06 & B C & 6 & -5 & \text {;VC1 }
\end{array} \\
& 14.9541 \\
& \text { 480.000E-06 } \\
& 0.4854770 \\
& 0.6000000 \\
& 10.3000 \\
& 309.000 \mathrm{E}-06 \\
& 5.0103 \\
& \begin{array}{lllll}
9 \text { MUL } & 8 & \multicolumn{1}{c}{\text {;PWM SWITCH }} \\
10 \mathrm{~L} & 9 & -12 & -11 & \text {;L2 }
\end{array} \\
& 11 \mathrm{R} \quad 10 \\
& 12 \mathrm{FIO} \quad 10 \\
& \operatorname{sen} \\
& \text {; } \mathrm{VL}=\mathrm{VC2}
\end{aligned}
$$

Fig. 18. The input format of the TUTSIM program.

entered directly to the TUTSIM simulation program. Users specify the transmittance and the inputs for each branch, define the injecting function of the duty-ratio, and give the initial condition, the simulation step size, and the starting and stopping times. The file to simulate the step response is shown in Fig. 18.

The circuit parameters are as follows: $V_{g}=15 \mathrm{~V}, f_{s}=$ $30 \mathrm{kHz}, L_{1}=0.43 \mathrm{mH}, L_{2}=0.48 \mathrm{mH}, C_{1}=31.4 \mu \mathrm{F}$, $C_{2}=30 \mu \mathrm{F}, R_{L 1}=0.25 \Omega, R_{L 2}=0.6 \Omega, R=10.4 \Omega$. An electronic switch was built to inject the duty-ratio step excitation at Point $A$. The Tektronix oscilloscope 2440 was used to measure the transient responses of the converter at Point $B$, for the output voltage $v_{o}$, and Point $C$, for the inductor current $i_{L 2}$. The single-shot mode was used, with the external signal from point $A$, to trigger the data acquisition to record the transients.

Experiment 1: For a large step duty-ratio jump, from $d=$ 0.355 to $d=0.69$, the output voltage jumped from $5 \mathrm{~V}$ to $9.7 \mathrm{~V}$, and the current jumped from $0.48 \mathrm{~A}$ to $0.93 \mathrm{~A}$. The measured data and the simulation data are plotted in the same figure as shown in Fig. 19. The measured large-signal response and the prediction match very closely.

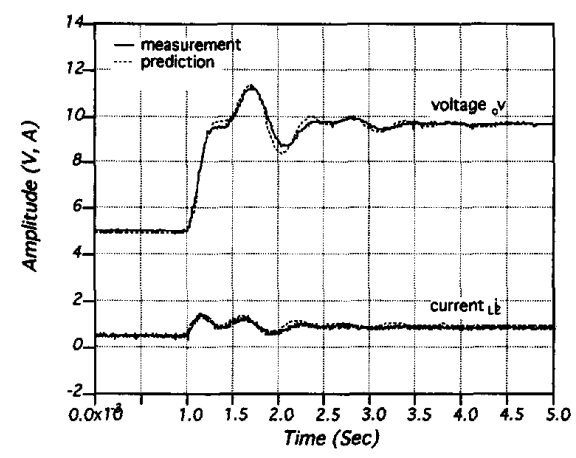

Fig. 19. The predicted and measured large-signal step response.

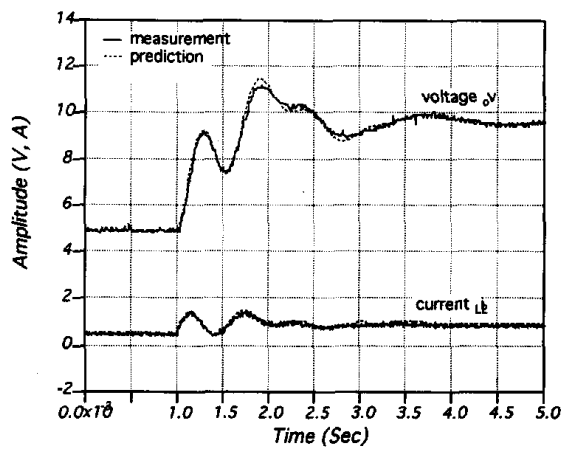

Fig. 20. The predicted and measured large-signal step response.

Experiment 2: The input inductor was changed from 0.43 mHto $1.45 \mathrm{mH}$, and other conditions remained the same as Experiment 3.2. The measured data and the simulation data are plotted together in Fig. 20. The prediction and the measurement are very close.

The experiments verified the Switching Flow-Graph largesignal model. This model can be utilized to predict the large-signal behavior of the PWM switching converters, and to provide global stability information.

\section{Small-Signal Model}

The small-signal linearized model plays an important role in the study of local small-signal dynamic behavior. For the buck converter with input filter, including the two parasitic resistors, the small-signal model is shown in Fig. 21. The control to output transfer function is derived from the Switching FlowGraph model:

$$
\begin{aligned}
\frac{\hat{v}_{o}}{d} & =\frac{K_{d}\left(1+\frac{1}{Q_{0} \omega_{0}} s+\frac{1}{\omega_{0}^{2}} s^{2}\right)}{D(s)} \\
D(s) & =\left(1+\frac{1}{Q_{1} \omega_{1}} s+\frac{1}{\omega_{1}^{2}} s^{2}\right)\left(1+\frac{1}{Q_{2} \omega_{2}} s+\frac{1}{\omega_{2}^{2}} s^{2}\right) \\
K_{d} & =\frac{V_{g}\left(1-\frac{D^{2} R_{L 1}}{R}\right)}{1+\frac{R_{L 2}+d^{2} R_{L 1}}{R}} \\
\omega_{0} & =\frac{1}{\sqrt{L_{1} C_{1}}}
\end{aligned}
$$




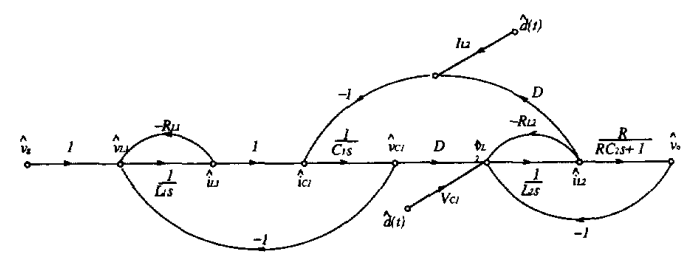

Fig. 21. The small-signal model of the buck converter with an input filter.

$$
\begin{aligned}
Q_{0} & =\frac{1}{R_{L 1} \sqrt{\frac{C_{1}}{L_{1}}}-\frac{D^{2}}{R} \sqrt{\frac{L_{1}}{C_{1}}}} \\
\omega_{1} & =\frac{1}{\sqrt{L_{1} C_{1}}} \\
Q_{1} & =\frac{R}{D^{2}} \sqrt{\frac{C_{1}}{L_{1}}} \\
\omega_{2} & =\frac{1}{\sqrt{L_{2} C_{2}}} \\
Q_{2} & =R \sqrt{\frac{C_{2}}{L_{2}}}
\end{aligned}
$$

Experiments were conducted to verify the prediction. The HP 3577 network analyzer was used to measure frequency response. A frequency sweeping signal was injected at Point $A$. The responses at Point $B$ were detected to determine the transfer function $\frac{\hat{v}_{0}}{\hat{i}}$. An IBM AT was connected to the analyzer through the HPIB interface to take the measurement data.

Experiment 3: A frequency sweeping signal, ranged from $5 \mathrm{~Hz}$ to $30 \mathrm{kHz}$, was injected at Point $A$. The responses at Point $B$ were detected to determine the transfer function $\frac{\hat{v}_{0}}{d}$. For the circuit parameter $V_{g}=15 \mathrm{~V}, f_{s}=30 \mathrm{kHz}, D=0.27$, $L_{1}=0.43 \mathrm{mH}, L_{2}=0.48 \mathrm{mH}, C_{1}=31.4 \mu \mathrm{F}, C_{2}=30 \mu \mathrm{F}$, $R_{L 1}=0.25 \Omega, R_{L 2}=0.6 \Omega, R=10.4 \Omega$. The model predicts two RHP zeros, when the duty-ratio $D>0.24$. The measured transfer function and the predicted transfer function are plotted in Fig. 22. The predicted and the measured response match closely. Both the prediction and the measurement show that the phase shifts $540^{\circ}$ at $2.4 \mathrm{kHz}$. The measurement proved the prediction.

The experiments proved that the large-signal Switching Flow-Graph model and the small-signal Switching FlowGraph model are very accurate.

\section{CONCLUSION}

The Switching Flow-Graph technique provides a unified graphical representation of the large-signal model, the smallsignal model, and the steady-state model for any given PWM switching converter. The graphic models are very easy to obtain and they yield a visual representation of switching converter systems. The large-signal model and the small-signal model are verified by experiments. The measurements and the theoretical predictions agree very well. This technique can be used to model very complicated switching converters, such as the coupled inductor converter, linear feedback systems, and nonlinear feedback systems, etc.
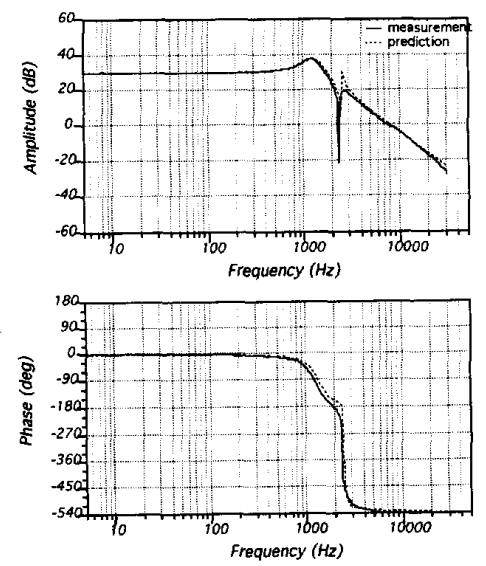

Fig. 22. The predicted and measured frequency response of $\frac{\hat{x}_{0}}{\hat{d}}$.

The modeling procedure is as follows: First, find the two subcircuits, the ON-circuit, when the switch is $\mathrm{ON}$, and the OFF-circuit, when the switch is OFF, for the given switching converter. Second, draw the flow-graphs for the two subcircuits and combine them using the switching branches. Then, replace the switching branches with their large-signal switch models to obtain the large-signal Switching FlowGraph, replace the switching branches with their small-signal switch models to obtain the small-signal Switching FlowGraph, or replace the switching branches with their steadystate switch models to obtain the steady-state Switching FlowGraph . Finally, the algebraic rules of the flow-graph can be used to simplify the small-signal model or the steady-state model.

The large-signal model is compatible with the TUTSIM simulation program. By simply entering flow-graph branches, the interconnections, the step size for the simulation, and the initial conditions, the program automatically generates the large-signal dynamic response. The large-signal model gives a global view of the system dynamics, which enables the designer to understand the limits of the system, and helps the designer to achieve robust control. The small-signal model provides analytic and graphic frequency response relations between each variable. This enables the designer to do smallsignal performance adjustments. These procedures may need to be repeated before a satisfactory closed-loop switching converter design is obtained. However, the approach using Switching Flow-Graph technique is faster, easier, and more robust than other modeling tools.

\section{REFERENCES}

[1] S. Ćuk and R. D. Middlebrook, "A general unified approach to modelling switching-converter power stages," IEEE Power Electronics Specialists Conference, 1976.

[2] R. Venkataramanan, A. Sabanovic, and S. Cuk, "Sliding mode control of dc-to-dc converter," International Conference on Industrial Electronics, Control and Instrumentations, 1985.

[3] R. W. Erickson, "Large signals modeling and analysis of switching regulators," IEEE Power Electronics Specialists Conference, 1982.

[4] B. C. Kuo, Automatic Control Systems. Englewood Cliffs, NJ: Prentice Hall; 1991.

[5] W. E. Reynolds and J. Wolf, TUTSIM User's Manual, 1987. 


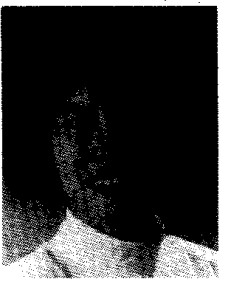

Keyue Smedley (S'87-M'90) received the B.S. and M.S. degrees in electrical engineering from Zhejiang University, China, in 1982 and 1985, respectively, and a Ph.D. degree in electrical engineering from the California Institute of Technology, in 1991.

Dr. Smedley was employed as an engineer at the Superconducting Super Collider from 1990 to 1992. She was responsible for determining the electrical characteristics of the superconducting magnet strings and designing the power conversion system for the collider. She has been a faculty member at the University of California, Irvine, since 1992. Her areas of interest include modeling and control of switching converters, digital-PWM of switching power amplifications, power factor corrections, and electrical characteristics of superconducting magnets. She currently holds one U.S. patent for onecycle control.

Dr. Smedley is a faculty member of Eta Kappa Nu.

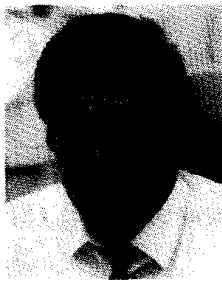

Slobodan Ćuk (M'74) received a B.S. degree from Belgrade University, Yugoslavia, in 1970 , a M.S degree from Santa Clara University, CA, in 1973, and a $\mathrm{Ph} . \mathrm{D}$. degree from the California Institute of Technology in 1976

Dr. Cuk is on full-time appointment as an Associate Professor of Electrical Engineering in the Division of Engineering and Applied Science at the California Institute of Technology. He is well known in the field of power conversion science for his contributions to modeling and analysis of dc-to-dc converts as well as for development of new magnetic structures for switching converts. For the invention of a new switching converter topology, now known as the ĆUkonverter ${ }^{\mathrm{TM}}$, and several of its extensions, he won a coveted IR 100 Award for 1980 from Industrial Research Magazine. For the invention of integrated magnetics and for expediting the development of switched-mode power converters, he obtained the 1991 Edward Longstreth Medal from the Franklin Institute. His publications include numerous papers in the power electronics field and three books on switched-mode power conversion. $\mathrm{He}$ holds four patents related to the new switching power conversion concept. Several patents are also pending. 